\title{
Setting up a Customized Electronic Health Record System Fit for Internal Medicine in Dakar, Senegal
}

\section{Baidy S. Y. Kane', Mamour Gueye ${ }^{2 *}$, Mohamed Dieng1, Atoumane Faye', Awa Cheikh Ndao1, Nafissatou Diagne ${ }^{1}$, Abdoulaye Pouye ${ }^{1}$}

${ }^{1}$ Department of Internal Medicine, Gynecologic and Obstetric Clinic, Dakar, Senegal

${ }^{2}$ Gynecologic and Obstetric Clinic, Aristide Le Dantec Teaching Hospital, Dakar, Senegal

Email: ^mamourmb@yahoo.fr

How to cite this paper: Kane, B.S.Y., Gueye, M., Dieng, M., Faye, A., Ndao, A.C., Diagne, N. and Pouye, A. (2019) Setting up a Customized Electronic Health Record System Fit for Internal Medicine in Dakar, Senegal. E-Health Telecommunication Systems and Networks, 8, 35-42.

https://doi.org/10.4236/etsn.2019.84004

Received: November 19, 2019

Accepted: December 28, 2019

Published: December 31, 2019

Copyright () 2019 by author(s) and Scientific Research Publishing Inc. This work is licensed under the Creative Commons Attribution International License (CC BY 4.0).

http://creativecommons.org/licenses/by/4.0/

(c) (i) Open Access

\begin{abstract}
Objective: Describe the design and implementation of an electronic medical record-E-INTMED-customized for Internal Medicine in Dakar, Senegal. Methodology: This study was carried out in a public teaching hospital in Dakar Senegal. It entailed collaboration between physicians specialized in various fields in Internal Medicine and Computer Scientists to carry out the compilation of data and their electronic transcription to produce a prototype which met users' needs. Results: E-INTMED software is structured around several hierarchical tables allowing users to register and store all relevant patients' information. E-INTMED structures patient's data to provide a clear overview of their medical history and users' activity performance. E-INTMED makes medical users' life so much easier. Users can generate and send letters and prescriptions quickly and efficiently using the customized templates which they can modify or create new ones. In addition to these capabilities, all of the features expected in an Internal Medicine EHR are handled by E-INTMED, such as lab orders and results, mechanisms for continuity of care, embedding and access to images and documents, and so much more. E-INTMED provides medical students with a number of educational, practical and administrative advantages. Conclusion: Computerization of medical records has become a necessity today. Crossing the line to Electronic medical records could help to improve medical practice and medical training.
\end{abstract}

\section{Keywords}

Internal Medicine, Electronic Medical Record, Dakar 


\section{Introduction}

The first known record is Egyptian, but it is not a proper patient record, rather a written document on papyrus describing surgical treatment of war wounds [1]. The notion of "patient record" appeared during the $14^{\text {th }}$ century. It allowed the doctors to remember the story of their patients. Then followed the Greeks with Hippocrates, the father of medicine, who wrote careful notes of his patients about symptoms, appearance of the patient, social situation and other parameters to decide on the treatment [2]. The Arabs introduced the concept of hospital. They were the first to keep written records of patients and their medical treatment, which were later edited by doctors and referenced in future treatment [3]. Later on, the first formal medical record system was developed in Sweden in 1752 and continuously refined until 1980 [2]. From 1970, nursing records have been part of medical records. The whole has become a communication and data transmission tool between healthcare professionals.

To keep, analyze and share information collected on everyday activity, databases are required, which necessarily implies computerization of the patient's record [4]. The electronic medical record was created in the 1990s, with the aim of improving the quality of care. Computing is not only a valuable aid for health care providers, it is at the heart of modern medicine, a medicine that requires information sharing between health system stakeholders and the patient in order to coordinate and sustain continuity of care. Almost all hospitals in developed countries have electronic databases, including names, addresses, diagnoses and treatments for all inpatients and outpatients. Investigators can acquire access to these databases to identify subjects for a study after gaining approval from a hospital's ethics committee [5].

Just from a chronological perspective, several decades separate developed countries to Africa.

Internal Medicine department plays the role of coordinating medical care, managing difficult diagnoses, systemic diseases and chronic diseases such as diabetes. To address these issues, our department, in Aristide Le Dantec teaching hospital, has completely been renovated in order to provide users with an environment that guarantees their comfort, safety and quality of care. On this background and considering advantages of Electronic medical record systems in health, the Internal Medicine department has evolved and initiated a computerization of medical records.

This paper describes the process and puts forward ways to maintain sustainability.

\section{Methods}

The study was carried out in a public teaching hospital in Dakar Senegal from January 2018 to December 2019. Aristide Le Dantec Hospital was created in 1912. This benchmark hospital gained acceptance since its set up in the management of complex medical and surgical health issues and also constitutes a 
learning platform for doctors, nurses and midwives either at undergraduate or post graduate level. Aristide Le Dantec has been labelled Level III hospital, the highest level in Senegalese health system with approximately 1200 health care providers. It hosts several medical and surgical departments including that of Internal Medicine. The latter plays the role of coordinating medical care, managing difficult diagnoses, systemic diseases and chronic diseases such as diabetes. The other departments include General surgery, Pediatrics, Cardiology, Obstetrics and Gynecology, Pathology, Oncology to name a few.

Five physicians specialized in various fields in Internal Medicine and Computer Scientists carried out the compilation of data and their electronic transcription. From January to August 2018, all stakeholders met on a regular basis at least fortnightly to review the content of each part of the database and agree on the relevant information to include in it. For each section, care was given to its electronic format for the sake of shareability and analysis.

As the process was going on, a prototype was generated and reviewed if required to meet users' needs until the final agreed database.

\section{Results}

\subsection{Description of E-INTMED}

E-INTMED consists of tables that are in relationship via foreign keys to the core table. It provides a solid foundation for users so that they can interact with the data stored in the structure. The interface mainly evolves around layouts and layout objects. E-INTMED interface was designed using web design tools and takes into account users' recommendations (physicians). The logic of the solution automates the tasks of users in order to increase their productivity. Calculations and scripts are the two main tools FileMaker Pro provides to automate the logic of the solution. Calculations provide access to complex and calculated data and require minimal interaction with users. Scripts allow users to perform tasks quickly and efficiently by automating parts of the process or by guiding users through the process. Scripts and calculations improve data integrity by standardizing processes. E-INTMED via FileMaker Pro provides tools for generating step-by-step reports, including attractive graphs to efficiently analyze and summarize data which can be sent by email, to Excel or printed out to PDF format to share with colleagues. To do this, E-INTMED combines data, an interface and the logic of a solution. One or more users may be accessing E-INTMED using FileMaker's deployment capabilities. Two types of storage are possible with E-INTMED. E-INTMED can be used as a local storage on a workstation or hosted on FileMaker Server on a local or remote server (hosting).

\subsection{Tables and Layouts}

E-INTMED structure is displayed in Figure 1.

\section{- Core table, general information}

Regarding the structure, we designed the core of the database to be the 


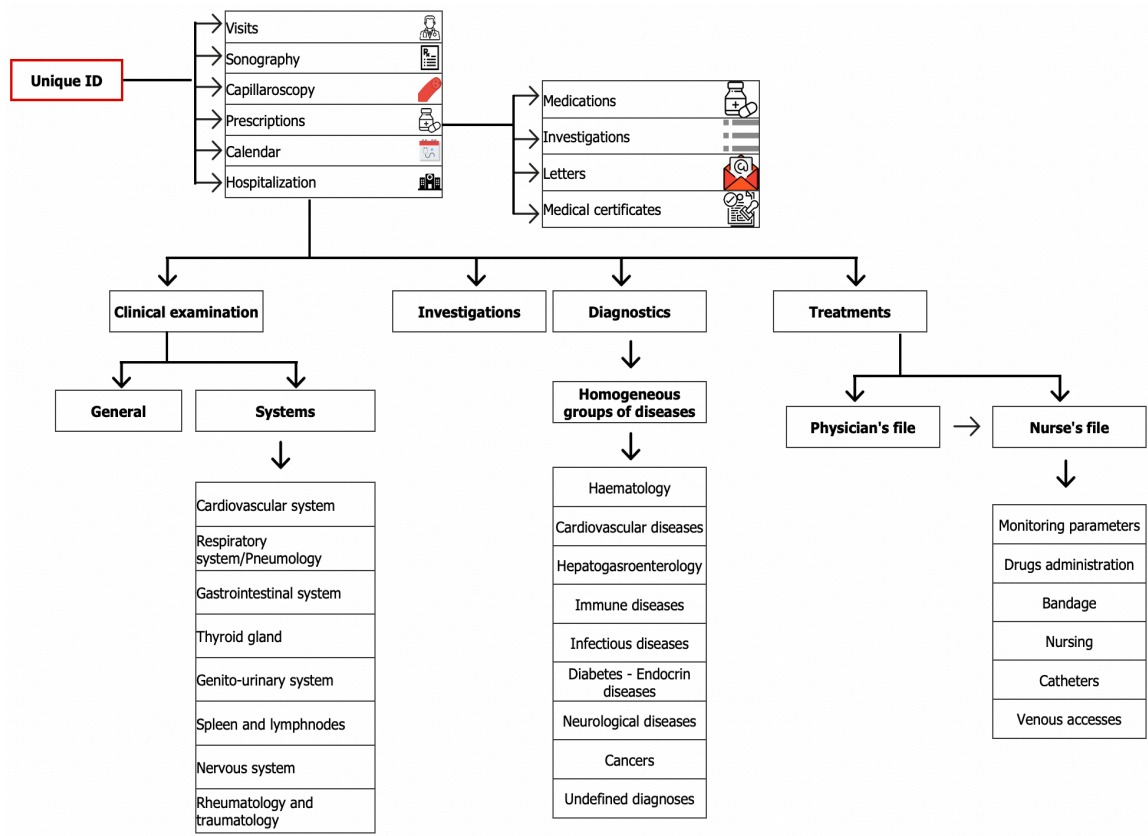

Figure 1. Structure of E-INTMED.

patient's General information table providing a primary key which populates related tables foreign keys. Demographic characteristics are stored within this table. The primary key is a 128-bit Universally Unique IDentifier (UUID) automatically generated by the software upon the creation of a new record. The UUID is unique. In some software packages, the probability that a UUID will be duplicated is not zero, but close enough to zero to be negligible. In ours, the system checks first a similar UUID before committing the new created; thus, the risk of duplicated UUID is zero.

Are recorded in this core table patient's name, date of birth, date of admission, addresses, telephone numbers, email addresses, occupation, marital status and other general information.

\section{- Medical history and visits tables}

Medical history as well as medical visits are recorded in separate related tables.

- Prescribing investigations, certificates, letters and prescriptions

The "Prescribe" table is made up of 4 items: medical prescriptions, investigation, letters and medical certificates. To help physicians in an easy way to perform their job and save writing time, E-INTMED provides them with several standard prescriptions that can be modified as needed. Others can be generated and stored.

Physicians can easily prescribe investigations from check boxes or drop drown menus. Furthermore, button bars are provided to automate prescriptions according to standard investigations. Prescriptions can also be sent to the patient by email. Medical prescriptions and letters follow the same process.

\section{- Hospitalization}

The Hospitalization table is an important part of E-INTMED. It comprises the 
dates of admission and discharge. Physical examination of all body systems is detailed in each matching section. Students, alone or accompanied by a supervisor, can assess the patient by following the guide from the software. Prescriptions are also displayed once ordered in the Nurse's file. The nurses are responsible for drug administration, which is displayed in a window with indicators of whether or not the treatment has been administered (Figure 2). If a diagnosis is made during or at the end of the hospitalization, this is recorded accordingly.

\section{- Gallery table}

Any media file (photo, audio, video) can be stored in the Gallery table and easily displayed, printed or shared with other physicians or with the patient.

\section{- Nurses' observation charts}

Components of a patient's nursing record include progress notes, medication charts, laboratory orders and reports, vital signs, observation charts, drug admission and patient's assessment forms.
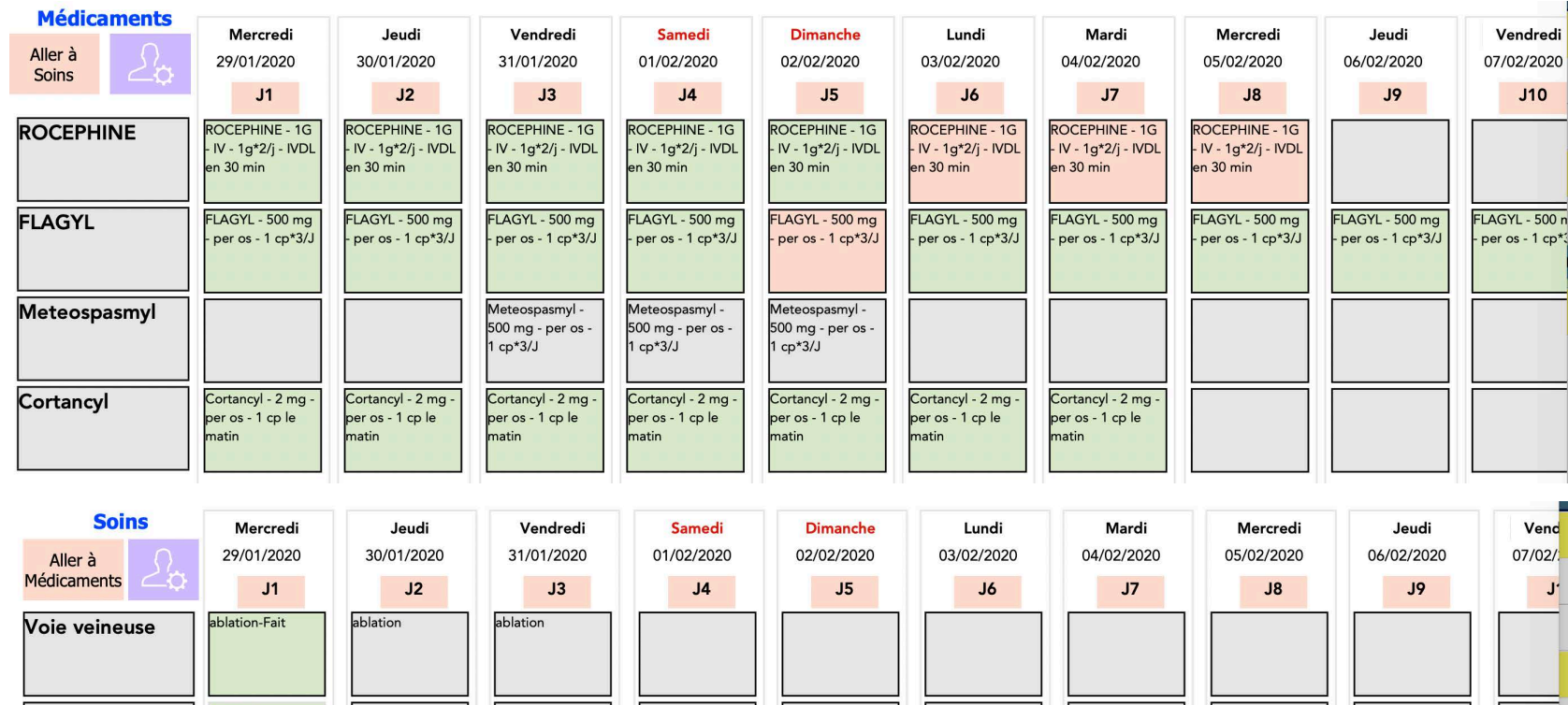

matin
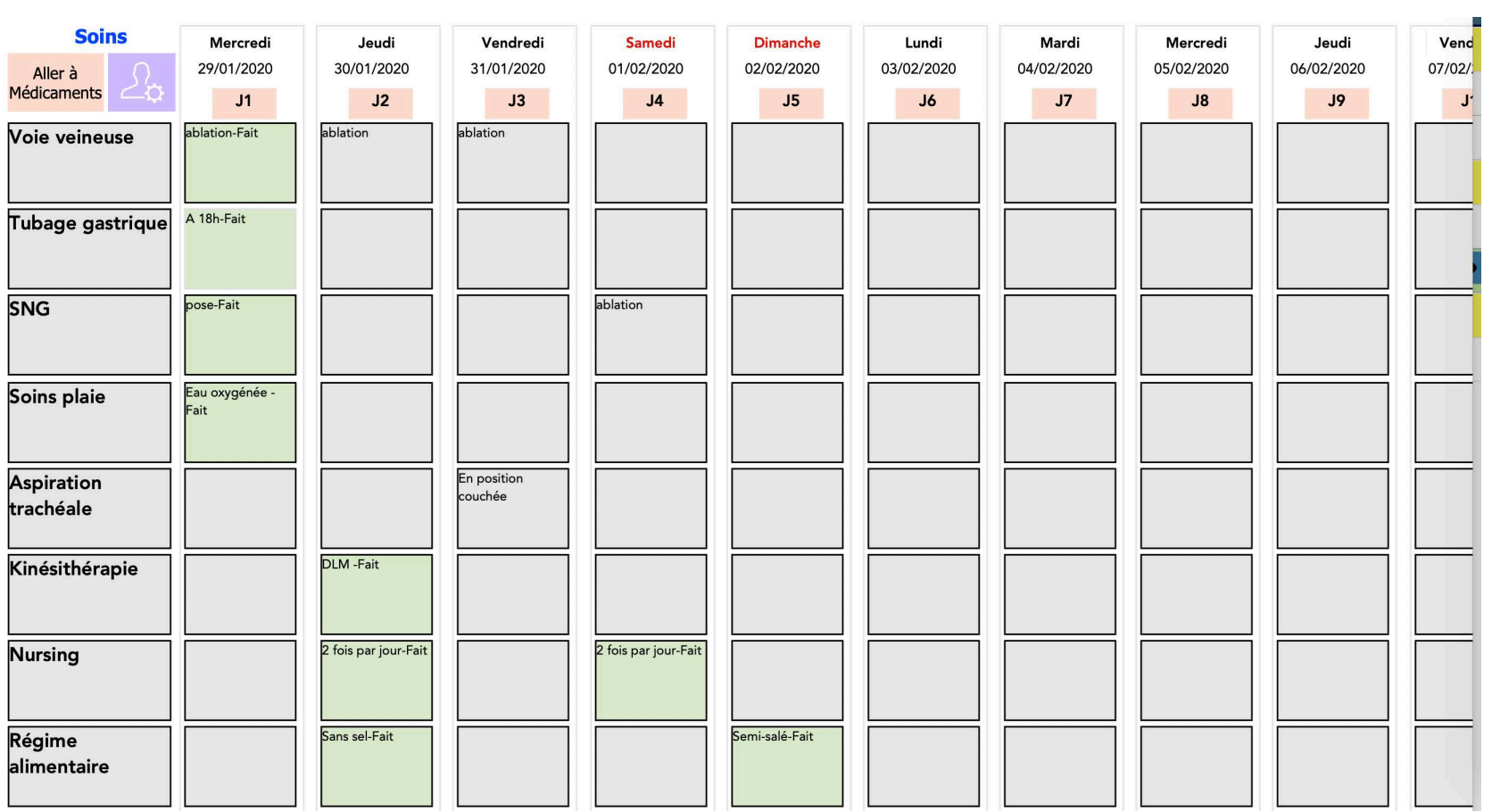

Figure 2. Chart displaying drug administration and nursing. 


\subsection{Tools}

\section{- Appointments}

Appointments are added into the database from any point and by any permitted user (secretaries, nurses, doctors). These can be modified or cancelled. Appointments are registered along with doctors' schedules and specialty.

Outputs sorted by doctors can be displayed, printed out or sent by mail in PDF format to doctors so they can be ready for their next day visits.

\section{- Requests and dynamic reports}

One of the main purposes of E-INTMED is to provide activity reports via requests. These allow physicians, board and health authorities to instantly be aware all the relevant epidemiological data. To go further, E-INTMED offers a real-time visual of the activity according to the chosen period. Each category allows to go much further in statistical analysis. Upon request, the whole medical file is easily displayed in a word (.doc) file which can subsequently be printed out or stowed.

\section{Discussion}

Several medical data management systems have been developed and are commercially available [6]-[13]. E-INTMED is a client-server electronic medical record (EMR) system created using FileMaker. FileMaker was chosen because it was the only application with the necessary set of tools: a client/server application that works in a wireless LAN, on Windows or Mac OS laptops, on iOS mobile devices (iPad and iPhone) and a physician who had expertise in FileMaker. FileMaker is a great, cost-effective tool for making databases. The system itself provides quick access to all patients' data, plan screenshots and medical documentation. Compared with other systems, we have found Filemaker to be easy to get started, easy to deploy locally, running on both platforms (Mac OS X, Windows, iOS) with many plugins available to extend functionality. Mobility and scalability are well taken into account allowing changing field/database/script names. Finally, it allows for importing excel, csv, tab-formatted sheets.

Additionally, the system allows a quick request on all the patient's medical information. Information is at hand during the follow-up examination and is accessible at any time.

Prospective data collection provides complete, accurate and valid data. The clearly organized and structured data entry and the continuous control scripts ensure the quality of data collection. Complex query scripts make it easy to retrieve large datasets. These datasets are processed for predefined statistical analyzes.

In addition to traditional learning resources such as lectures and textbooks, students are increasingly using e-learning tools like commercially available question banks to supplement their learning. E-INTEMED allows students to put into practice what they've learnt by using a tool that walk them through step by step patient's physical assessment. The EHR has changed how medicine is practiced and student experiences with the EHR early-on are likely to have a long-term impact. However, medical students along with other users need to be well trained 
to use efficiently the software. Chi et al. noted that medical students at their institution spent $4.42(3.00$ - 6.17) hours per day interacting with their EHR, primarily focused upon chart review rather than documentation or order entry. They pointed out that, without guidance, the EHR can lead to unintended consequences and students would likely benefit from consistent observation and a structured curriculum for proper use [14]. Further research is required to evaluate the impact of student-centered EHR systems on learning outcomes.

Electronic medical record data represent a rich resource for clinical research. Several Electronic medical records are, however, collected for the purposes of clinical documentation and billing rather than research. This fact creates obstacles to their use in scientific investigation. Particular issues include accuracy, completeness, comparability between settings, ease of extraction, and context of recording [15]. To avert this issue, clinicians and IT worked together during the whole process. To perform epidemiological studies, data can be imported into Excel or SPSS (Statistical Package for Social Science) for further statistical analysis.

Despite the growing literature on benefits of various EHR functionalities, some authors have identified potential disadvantages associated with this technology. These include financial issues, changes in workflow, temporary loss of productivity associated with EHR adoption, privacy and security concerns, and several unintended consequences [16].

\section{Conclusions}

This platform provides practitioners with a suitable tool with secure access which enables information to be entered; to ensure a "real time" control of the prescriptions and the management of the lab results.

It also makes available to medical students, during their internship, an interactive tool for learning practical semiology and drawing medical observation files.

Above all, it provides a secure computer archiving system for quality of care and clinical research. This project could be extended to other medical services.

We will continue to improve E-INTMED and work toward simplifying it with the goal of remaining paperless, streamlining visits, processes, and reducing operating costs for our clients.

\section{Authors' Contribution}

All authors substantially contributed to the conception, design, acquisition, and interpretation of data. All authors have also participated in drafting and revising the manuscript and approved the final version.

\section{Conflicts of Interest}

The authors declare no conflicts of interest regarding the publication of this paper.

\section{References}

[1] Al-Awqati, Q. (2006) How to Write a Case Report: Lessons from 1600 B.C. Kidney 
International, 69, 2113-2114. https://doi.org/10.1038/sj.ki.5001592

[2] Dalianis, H. (2018) Clinical Text Mining: Secondary Use of Electronic Patient Records. Springer, Cham. https://doi.org/10.1007/978-3-319-78503-5

[3] Miller, A.C. (2006) Jundi-Shapur, Bimaristans, and the Rise of Academic Medical Centres. Journal of the Royal Society of Medicine, 99, 615-617. https://doi.org/10.1177/014107680609901208

[4] Guèye, M., Ndiaye Guèye, M.D., Mbaye, M., Abdoulaye, M., Diouf, A., Wade, M., et al. (2017) Crossing the Line to Electronic Medical Records in Subsaharian Africa: An Obstetric and Neonatal Information System with Perinatal Indicators Dashboard. Journal of Health Science Research, 2, 14-20. https://doi.org/10.18311/jhsr/2017/15932

[5] Aschengrau, A. and Seage, G.R. (2020) Essentials of Epidemiology in Public Health. Jones \& Bartlett Learning, Burlington, MA, 237-266.

[6] Benjamin, I., Noumoff, J.S., Carlson Jr., J.A., Giuntoli, R.L., Morgan, M. and Mikuta, J.J. (1990) Database Management for a Gynecologic Oncology Service. Gynecologic Oncology, 38, 431-436. https://doi.org/10.1016/0090-8258(90)90086-Z

[7] Raab, G. and van Den Bergh, M. (2001) Development and Integration of the Oncological Documentation System ODS. Zentralblatt für Gynäkologie, 123, 444-449. https://doi.org/10.1055/s-2001-17241

[8] Salenius, S.A., Margolese-Malin, L., Tepper, J.E., Rosenman, J., Varia, M. and Hodge, L. (1992) An Electronic Medical Record System with Direct Data-Entry and Research Capabilities. International Journal of Radiation Oncology, Biology, Physics, 24, 369-376. https://doi.org/10.1016/0360-3016(92)90693-C

[9] Jacob, R. and Welkoborsky, H.J. (2002) Presentation of an Oncological Database Adapted for Head and Neck Cancer. Laryngorhinootologie, 81, 875-881.

https://doi.org/10.1055/s-2002-36104

[10] Poorten, V.V., Hart, A., Vauterin, T., Jeunen, G., Schoenaers, J., Hamoir, M., et al. (2009) Prognostic Index for Patients with Parotid Carcinoma: International External Validation in a Belgian-German Database. Cancer, 15, 540-550. https://doi.org/10.1002/cncr.24015

[11] Mira, E., Lanza, L., Castelli, A., Benazzo, M. and Tinelli, C. (1998) A Computerized Database for Managing Otorhinolaryngologic Oncology Patients. ACTA Otorhinolaryngologica Italica, 18, 155-163.

[12] Landis, S.H., Murray, T., Bolden, S. and Wingo, P.A. (1999) Cancer Statistics. $C A: A$ Cancer Journal for Clinicians, 49, 8-31. https://doi.org/10.3322/canjclin.49.1.8

[13] Wagenblast, J., Adunka, O., Gstottner, W., Arnoldner, C., Riedl, N., Diensthuber, M., et al. (2010) AdOnco Database-Six Years' Experience with the Documentation of Clinical and Scientific Data on Patients with Head and Neck Cancer. In Vivo, 24, 603-606.

[14] Chi, J., Bentley, J., Kugler, J., and Chen, J.H. (2019) How Are Medical Students Using the Electronic Health Record (EHR)?: An Analysis of EHR Use on an Inpatient Medicine Rotation. PLoS ONE, 14, e0221300. https://doi.org/10.1371/journal.pone.0221300

[15] Wasserman, R.C. (2011) Electronic Medical Records (EMRs), Epidemiology, and Epistemology: Reflections on EMRs and Future Pediatric Clinical Research. Academic Pediatrics, 11, 280-287. https://doi.org/10.1016/j.acap.2011.02.007

[16] Menachemi, N. and Collum, T.H. (2011) Benefits and Drawbacks of Electronic Health Record Systems. Risk Management and Healthcare Policy, 4, 47-55. https://doi.org/10.2147/RMHP.S12985 